(C) Masson, Paris, 1980.

\title{
Evolution des granulomes hépathiques dans différents types d'infections répétées à Schistosoma mansoni chez la souris.
}

\author{
par A. A. ABOUZKHAM et A. BUTTNER \\ Laboratoire de Parasitologie, U.E.R. des Cordeliers \\ 15, rue de l'Ecole-de-Médecine, 75006 Paris
}

\begin{abstract}
RESUME. Les effets observés sur les granulomes hépatiques chez des souris suisses albinos après des infections répétées à $S$. mansoni sont analysés dans ce travail: le temps écoulé entre la première et la deuxième infection a une influence majeure sur le nombre, la taille et les caractères histologiques des granulomes dans les infections résiduelles. Un délai de 15 jours entre les 2 infections ne stimule aucune protection et la deuxième infection se développe sans contrainte; un délai de 6 mois fait échec à une réinfection même sévère. La maturité des vers de première infection, et le dépôt des œufs dans les tissus paraissent nécessaires pour que s’instaure une immunité protectrice. Toutefois, au-dessous d'un certain taux d'infection primaire (50 cercaires), aucune protection n'a pu être obtenue malgré un délai de 6 mois écoulé entre les 2 infections; tandis que cette protection devenait effective quand le taux de primo-infection atteignait 100 ou 200 cercaires. Le rôle stimulateur d'un taux élevé de première infection est confirmé par certains auteurs, infirmé par d'autres. Il semble, comme le présument Smith et Clegg (1979), que les niveaux différents d'immunité chez la souris soient principalement liés à l'hétérogénéité des cercaires infestantes, et sans doute à leur taux moyen d'antigénicité.
\end{abstract}

Evolution of hepatic periovular granulomas in different types of repeated infections with $\mathrm{S}$. mansoni in mice.

SUMMARY. Effects upon hepatic granulomas in Swiss albino mice after challenged exposures to S. mansoni are studied : the time between the previous and the challenged infections had a major influence on the number, size and histological features of gra-

Accepté le 15 janvier 1980. 
nulomas in residual infections; 15 days delay has not stimulated any protection against the challenge which developed freely, while 6 months delay impeded the 2nd infection which was abortive. However, in spite of a 6 months delay observance, no stimula. tion of protective immunity was obtained when the level of the primary infection was of 50 cercariae, while this protection became effective when the rate of primo-infection reached 100 or 200 cercariae. Opinions differ on the part of primo-infection in the induction of a protective immunity. It seems that the rate of efficiency of primoinfection depends on the mean level of antigenicity of the infective cercariae.

\section{Introduction}

La formation des granulomes autour des œufs de $S$. mansoni déposés dans le foie est, on le sait, l'un des facteurs déterminants de l'hépatosplénie bilharzienne ; c'est à ce niveau que nous nous sommes placés pour étudier les effets histopathologiques produits par des infestations successives dont les taux d'intensité et les intervalles de temps qui les séparent ont été modulés.

La souche de $S$. mansoni utilisée pour ces expériences, conservée en laboratoire depuis plusieurs années, est entretenue régulièrement par passages sur Biomphalaria glabrata et sur des souris Swiss albino non consanguines.

Les hôtes, prélevés au hasard parmi ces souris, dans les mêmes tranches d'âge, ont le défaut de masquer les facteurs individuels de sensibilité ou de résistance existant à l'intérieur de l'espèce, ce qui limite la portée de nos observations. Mais ce choix avait pour objet de rechercher si, dans une population naturelle, vivant dans un lieu donné et fréquentant un gîte où le complexe Mollusque-Schistosome est apparemment stable, des individus de même maturité réagissaient de façon comparable lorsqu'ils étaient soumis à des conditions identiques de réinfestation.

Les souris parasitées par l'espèce $S$. mansoni ont l'avantage de présenter des lésions hépatiques assez semblables à celles décrites dans l'hépatite bilharzienne humaine, parallélisme d'autant plus remarquable que l'infection est légère et qu'elle est unique (la pathologie hépatique d'une souris exposée à un seul couple de vers est à cet égard significative: Andrade et Warren, 1964). Mais les réinfections posent le problème de la protection apportée par la primo-infection. Cette protection nous n'avons pu l'obtenir, dans une population ouverte, qu'en pratiquant des taux élevés de primoinfection, certains voisins de la léthalité. La proportion des individus survivants s'est néanmoins révélée suffisante, dans les différents cas envisagés, pour constater une certaine homogénéité des résultats dans les modalités de la protection. 


\section{Matériel et Méthodes}

Quatre vingt dix souris adultes de 18 à $20 \mathrm{~g}$ ont été infestées par immersion selon la méthode de Peres et Warren (1969). Les différents taux d'infection, obtenus par la technique d'Olivier et Stirewalt (1962), ont été pour la première infection de 50, 100 et 200 cercaires, pour la deuxième de 50 ou de 200 cercaires.

Entre les 2 infections, des intervalles de temps progressivement croissants ont été appliqués : 2,6 et 24 semaines.

Les foies, prélevés à l'autopsie, ont été placés pendant 48 heures dans le fixateur aqueux de Bouin; après inclusion à la paraffine, des coupes sériées ont été pratiquées pour suivre le développement des lésions hépatiques produites par les œufs : coupes de $5 \mu$ aux stades inflammatoires, de 7,5 $\mu$ pour les foies en voie de sclérose. Les colorations ont également tenté d'objectiver cette évolution anatomo-pathologique : coloration classique à l'hémalun-éosine, assez favorable à l'étude cytologique des poussées inflammatoires; Azan de Heidenhain, mettant en évidence l'état pathologique du parenchyme hépatique : coloration jaune et grise du cytoplasme normal ou en voie de régénération; coloration bleue du collagène, des pseudo-tubercules et des travées fibreuses.

Tableau I. Evolution de l'Infection témoin

\begin{tabular}{|c|c|c|c|c|}
\hline \multirow{2}{*}{$\begin{array}{l}\text { Nombre } \\
\text { de souris }\end{array}$} & \multirow{2}{*}{$\begin{array}{c}\text { Durée } \\
\text { de l'infection } \\
\text { (en semaines) }\end{array}$} & \multicolumn{2}{|c|}{$\begin{array}{c}\text { Granulomes } \\
\text { (moyenne par champ 10) }\end{array}$} & \multirow{2}{*}{ Ecart-type } \\
\hline & & Nombre & Taille $($ en $\mu)$ & \\
\hline 1 & 6 & 11,25 & 392 & 1,50 \\
\hline 2 & 12 & 26 & 272 & 2,29 \\
\hline 3 & 24 & 32,25 & 152 & 3,86 \\
\hline 5 & 48 & 22,25 & 112 & 2,42 \\
\hline 3 & 72 & 10,73 & 48 & 1,26 \\
\hline
\end{tabular}

Pour fournir une expression quantitative des modifications chronologiques des granulomes et des effets de l'hypersensibilité retardée (Warren et coll., 1967), les indices moyens du nombre et de la taille des granulomes ont été mesurés, puis statistiquement comparés en fonction de la durée des infections (Infections témoins: tableau I. Infections résiduelles (1) et infections témoins: tableau III). Ce calcul, effectué dans chaque cas sur 100 granuolomes, a été pratiqué à l'objectif 10 et aux

(1) Après réinfestation. 
micromètres objectif et oculaire. Seuls les granulomes entourant des œufs vivants ont été retenus. Pour déterminer la taille de chaque granulome, selon la technique de von Lichtenberg (1962), 2 diamètres ont été considérés: le plus grand $\mathrm{D}_{1}$ et son diamètre perpendiculaire $\mathrm{D}_{2}$; c'est leur moyenne arithmétique qui figure sur nos statistiques. Le nombre moyen des œufs demeurés vivants (permettant d'évaluer le taux de destruction des œufs) a été calculé dans les mêmes conditions.

\section{Protocoles d'Infestation}

Une infestation simple, témoin, a été effectuée avec 200 cercaires sur 30 souris ; 14 individus ont survécu, sacrifiés à des stades différents de leur infection. Le tableau I rend compte du nombre et du diamètre moyens des granulomes à ces différents stades.

Les infections répétées ont été pratiquées sur 4 lots inégaux de souris $(20,20$, $10,10)$ dont les survivants $(\mathrm{Sa}, \mathrm{Sb}, \mathrm{Sc}, \mathrm{Sd})$ sont respectivement de $8,7,3$ et 3 . Les conditions de primo-infection, de réinfection et d'autopsie, sont rapportées dans le tableau II.

Tableau II. Protocoles d'expérimentation

\begin{tabular}{|ccccc|}
\hline Lots souris & \multicolumn{2}{c}{ Taux d'infection } & $\begin{array}{c}\text { Intervalle } \\
\mathrm{I}_{1} / \mathrm{I}_{2} \\
\text { (en semaines) }\end{array}$ & $\begin{array}{c}\text { Duré IR } \\
\text { à l'autopsie } \\
\text { (en semaines) }\end{array}$ \\
\cline { 2 - 3 } & $\mathrm{I}_{1}$ & $\mathrm{I}_{2}$ & 2 & 6 \\
\hline $2 \mathrm{Sa}$ & 200 & 50 & 2 & 8 \\
\hline $3 \mathrm{Sa}$ & 200 & 50 & 2 & 12 \\
\hline $3 \mathrm{Sa}$ & 200 & 50 & 6 & 12 \\
\hline $2 \mathrm{Sb}$ & 200 & 50 & 6 & 24 \\
\hline $5 \mathrm{Sb}$ & 200 & 50 & 24 & 32 \\
\hline $3 \mathrm{Sc}$ & 100 & 200 & 24 & 32 \\
\hline $3 \mathrm{Sd}$ & 50 & 50 & & \\
\hline
\end{tabular}

$I_{1}$ : Primo-infection; $-I_{2}$ : Deuxième infection; $-I R$ : Infection résiduelle.

\section{Résultats}

Dans une infection simple, on constate une évolution favorable des granulomes hépatiques dont le diamètre régresse à partir de la $12^{\mathrm{c}}$ semaine ; les sujets qui atteignent la $72^{\mathrm{e}}$ semaine présentent une diminution sensible à la fois du nombre et de la taille des granulomes (tableau I) ; la nature histologique de la lésion s'est également modi- 
fiée : les anciens granulomes sont denses, fibreux, les œufs pratiquement détruits ; les néo-granulomes constitués de macrophages, mononucléaires et lymphocytes sont rares, petits et circonscrits.

Dans les infections répétées, le nombre et la taille des granulomes à l'autopsie sont significatifs : comparés à ceux d'une infection simple, témoin, de même durée, ils font apparaître le rôle du temps écoulé entre les deux infections et celui du taux de la première infection dans l'établissement d'une immunité protectrice.

\section{A. - Rôle du temps écoulé (tableaux II et III).}

Dans le lot Sa, l'intervalle $I_{1} / I_{2}$ est de 2 semaines, l'autopsie étant pratiquée 4 , 6 et 10 semaines après la deuxième infection : le nombre des granulomes résiduels est toujours supérieur à celui de l'infection simple, témoignant du succès de la deuxième infection et de l'absence d'immunité préventive; le diamètre moyen traduit peut-être une certaine accentuation des processus de blocage de l'hypersensibilité retardée, mais le taux de destruction des œufs (1) ne paraît pas affecté par une sensibilisation antérieure (tableau III).

Dans le lot $\mathrm{Sb}$, l'intervalle $\mathrm{I}_{1} / \mathrm{I}_{2}$ est de 6 semaines, l'autopsie étant pratiquée 6 et 18 semaines après la deuxième infection : les indices moyens du nombre et du diamètre des granulomes résiduels, du taux de destruction des œufs sont chacun inférieurs à la

Tableau III. Résultats comparés de l'Infection résiduelle et l'Infection témoin

\begin{tabular}{|c|c|c|c|c|c|c|c|c|c|c|}
\hline \multirow{3}{*}{$\begin{array}{l}\text { Lots } \\
\text { souris }\end{array}$} & \multirow{3}{*}{$\begin{array}{c}\text { IR } \\
\text { (en } \\
\text { semai- } \\
\text { nes) }\end{array}$} & \multicolumn{6}{|c|}{ Granulomes (moyenne par $\times 10$ ) } & \multirow{2}{*}{\multicolumn{3}{|c|}{$\begin{array}{l}\text { Nombre moyen } \\
\text { d'œufs vivants }\end{array}$}} \\
\hline & & \multicolumn{3}{|c|}{ Nombre } & \multicolumn{3}{|c|}{ Diamètre $(e n \mu)$} & & & \\
\hline & & IR & IT & $\Sigma_{1}$ & IR & IT & $\Sigma_{2}$ & IR & IT & $\Sigma_{3}$ \\
\hline $2 \mathrm{Sa}$ & 6 & 15 & 11,25 & 2,01 & 348,8 & 392 & 1,59 & 10 & 12 & 2 \\
\hline $3 \mathrm{Sa}$ & 8 & 17,5 & 12,5 & 2,1 & 368 & 362 & 0,3 & 13,8 & 20 & 2,8 \\
\hline $3 \mathrm{Sa}$ & 12 & 31 & 26 & 2,2 & 240 & 272 & 1,63 & 5,8 & 8 & 2,4 \\
\hline $2 \mathrm{Sb}$ & 12 & 21,65 & 26 & 0,68 & 304 & 272 & 1,17 & 7,4 & 8 & 0,76 \\
\hline $5 \mathrm{Sb}$ & 24 & 23,67 & 32,25 & 1,59 & 176 & 152 & 1,59 & 3,6 & 4,4 & 0,5 \\
\hline $3 \mathrm{Sc}$ & 32 & 25,5 & 26,25 & 0,33 & 168 & 152 & 0,92 & 2,2 & 1,6 & 0,27 \\
\hline $3 \mathrm{Sd}$ & 32 & 37,5 & 26,25 & 2,8 & 232 & 152 & 9,16 & 8,8 & 1,6 & 4,3 \\
\hline
\end{tabular}

IT : Infection témoin. - IR : Infection résiduelle. $-\Sigma$ : Ecart réduit $\mathrm{I}_{1} / \mathrm{IR}$. $\Sigma \geqslant$ : pas de protection.

(1) Taux apprécié d’après le nombre d'œufs restés vivants dans les granulomes. 
variation standard $(1,96)$ (tableau $I I I)$; au point de vue histologique, tous les bilharziomes, même à 6 semaines de la deuxième infection, sont à un stade largement chronique et doivent donc appartenir à la primo-infection : la protection due à l'infection antérieure paraît évidente.

Dans le lot Sc, l'intervalle $\mathrm{I}_{1} / \mathrm{I}_{2}$ est de 24 semaines, l'autopsie étant pratiquée 8 semaines après la deuxième infection : le nombre et le diamètre moyens des granulomes résiduels demeurent comparables à ceux de l'infection témoin (tableau III). L'aspect histologique du foie est celui d'une hépatite bilharzienne chronique ancienne. La protection semble patente et d'autant plus affirmée que la deuxième infection était de 200 et non de 50 cercaires.

\section{B. - Rôle du taux de la première infection (tableaux II et III).}

Dans le lot Sd, l'intervalle $\mathrm{I}_{1} / \mathrm{I}_{2}$ est toujours de 24 semaines, l'autopsie étant pratiquée 8 semaines après la deuxième infection; mais le taux de la première infection a été ramené à 50 cercaires: d'après les 3 paramètres étudiés, la deuxième infection évolue au niveau du foie sans qu'aucun processus antérieur de défense paraisse entraver son développement (Nombre: $\Sigma=2,8$; diamètre : $\Sigma=9,16$; œufs vivants : $\Sigma=4,3$ ) (tableau III). La déviation standard confirme donc chaque fois l'absence de protection. L'aspect évolutif des bilharziomes est également révélateur : les uns sont fibreux, anciens, les autres granulomateux, jeunes et florides. La concomittance des 2 infections ne semble pas faire de doute.

Si l'on se reporte aux expériences antérieures où les taux de primo-infection étaient de 100 ou de 200 cercaires, force est de constater qu'indépendamment du temps écoulé entre les 2 infections, l’impulsion donnée par la primo-infection paraît nécessaire pour que se déclenchent les mécanismes d’immuno-protection.

\section{Commentaires et conclusion}

Les effets observés dans cette série d'expériences mettent en évidence la nécessité de respecter un certain intervalle de temps entre 2 infections successives pour que s'instaurent contre la deuxième infection des moyens de prévention appréciables au niveau des granulomes hépatiques.

Dès 1974, Sher et coll. avaient démontré que diverses souches de souris développaient régulièrement, après une première infection à $S$. mansoni, une résistance contre une réinfection homologue lorsque celle-ci était pratiquée au moins 6 semaines après la primo-infection; quand ce délai atteignait 12 à 16 semaines, cette protection devenait substantielle: mesurée au niveau du poumon, elle accusait, par rapport aux témoins, une chute de 50 à $75 \%$ des schistosomules chez les souris sensibilisées. Pour ces auteurs, une immunité vraie ne peut être induite que si les parasites de la première infection sont adultes et entièrement développés. Long et coll. (1978) aboutissent à la même constatation dans des expériences de réinfections à $S$. mansoni où 
ils n'ont obtenu une protection sur les plans humoral et cellulaire que lorsque la maturité des vers de première infection était acquise et leurs œufs déposés dans les tissus.

Il était dès lors prévisible que 2 semaines après la première infection de nos souris $\mathrm{Sa}$, les schistosomes encore immatures ne pourraient induire une immunité véritable contre une nouvelle infection. A ce stade, Sher et coll. ne leur reconnaissent que la faculté de provoquer un certain retard dans la migration des schistosomules de deuxième infection vers les poumons, non celle de les détruire. Au niveau du foie, en effet, le nombre des granulomes du lot $\mathrm{Sa}$, toujours supérieur à celui des souris témoins, attestait le succès de la deuxième infection.

A 6 semaines de la première infection, les vers étaient devenus adultes et une barrière immunitaire pouvait faire obstacle à une deuxième infection. C'est ce qu'ont confirmé les 3 paramètres étudiés et l'aspect histologique des granulomes hépatiques résiduels du lot $\mathrm{Sb}$. A 24 semaines de la première infection, cette protection s'était maintenue et même renforcée puisqu'elle faisait échec à une réinfection de 200 cercaires chez les souris Sc.

Cette immunité paraît persister assez longtemps puisqu'elle était encore observée 12 mois après la première infection de souris Swiss albino par Warren et coll. (1977).

En dehors de la nécessaire maturité de la première infection pour que la seconde devienne abortive, la question du rôle du seuil de cette primo-infection reste très controversée : avec un taux initial abaissé de 200 à 50 cercaires, et malgré le long délai observé entre les 2 infections ( 6 mois), nous n'avons obtenu aucune immunité chez les 3 exemplaires de notre lot $\mathrm{Sd}$. Ce phénomène a été confirmé par divers auteurs : Ritchie et coll., 1966 ; Foster et Broomfield, 1971; Long et coll., 1978, bien que ces derniers aient obtenu une résistance partielle avec des taux initiaux de 25 et 35 cercaires. Plus récemment, dans des infections doubles à $S$. haematobium et S. mansoni, Michaël et coll., 1979 ont constaté la taille réduite des granulomes de deuxième infection de $S$. mansoni ; ils admettent qu'en raison de l'identité de structure antigénique des 2 schistosomes (Capron et coll., 1966), une réaction d'immunité croisée a supprimé les effets immunopathologiques de la deuxième infection; mais, la primo-infection était de 200 cercaires, et on peut se demander si un taux initial plus bas aurait suffi à stimuler cette immunité protectrice.

Dans un sens très différent, Sher et coll. (1974) ont régulièrement observé des destructions importantes de schistosomules de deuxième infection dans les poumons avec des taux très bas de première infection ( 3 à 20 schistosomes adultes). Ils concluent que ces taux de destruction ne dépendent pas du niveau de la première infection.

Cette troublante disparité des niveaux d'immunité dans les réinfestations a été particulièrement étudiée par Smith et Clegg (1979) : dans une série d'expériences, ils observent chez diverses souches de souris, consanguines ou non, des niveaux très variables d'immunité contre une deuxième infection, même lorsqu'elle est effectuée 12 à 15 semaines après la première. La variabilité est moindre lorsque les cercaires utilisées pour les 2 infections sont de même origine que lorsqu'elles proviennent de 2 sources différentes. Des recherches plus précises sur une seule souche de souris 
montrent que des cercaires de gîtes différents induisent des niveaux variables d'immunité contre une réinfestation issue d'un même gîte ; tandis que des réinfestations d'origines différentes témoignent de degrés variables de sensibilité à l'immunité stimulée par une infection primaire émanant d'une même source. L'isolement de clones cercariens à partir d'un seul miracidium révèle que ces clones eux-mêmes opposent des sensibilités différentes à l'immunité induite par une même infection primaire. Les auteurs déduisent de ces observations que les niveaux d'immunité ou de résistance sont davantage liés à l'hétérogénéité des cercaires qu'à celle des hôtes utilisés.

Dans ces conditions, peut-être les taux variables de primo-infection susceptibles de stimuler une immunité protectrice contre une réinfection dépendent-ils, en dernière analyse, des niveaux moyens d'antigénicité et de résistance des cercaires des 2 infections.

\section{Bibliographie}

Abouzkham A.A.: Modulation de la pathologie hépatique chez la souris dans différents types de pluri-infestations à Schistosoma mansoni. Mémoire. Université de Paris VII, 1979.

Andrade Z.-A., Warren K.S.: Mild prolonged schistosomiasis in mice. Alterations in host response with time and the development of portal fibrosis. Trans. Roy. Soc. Trop. Med. Hyg., 1964, 58,53 .

Block E.H., Abdel Wahab M.F., Warren K.S.: In vivo microscopic observations of the pathogenesis and patho-physiology of hepato-splenic Schistosomiasis in the mouse liver. Amer. J. Trop. Med. Hyg., 1972, 21, 5, 546.

Boros D. L., Pelley R.P., Warren K. S.: Spontaneous modulation of granulomatus hypersensitivity in Schistosomiasis mansoni. J. Immun., 1975, 114, 5, 1437-1441.

Capron A., Vernes A., Biguet J., Rose F., Clay A.: Les précipitines sériques dans les Bilharzioses humaines et expérimentales à Schistosoma mansoni, Schistosoma haematobium et Schistosoma japonicum. Ann. Parasitol. Hum. Comp., 1966, 41, 123.

Cheever A.W.: A comparative study of Schistosoma mansoni infections in mice, gerbils, multimammate rats and hamsters. I. The relation of portal hypertension to size of hepatic granulomes. Am. J. Trop. Med. Hyg., 1965, 14, 211.

Doenhoff M., Bickle Q., Long E., Bain J., Mc Gregor A.: Factors affecting the acquisition of resistance against Schistosoma mansoni in the mouse. I. Demonstration of resistance to re-infection using a model system that involves perfusion of mice within three weeks of challenge. J. Helm., 1978, 52, 173-186.

Foster R., Broomfield K.E.: Preliminary studies on the development of Schistosoma mansoni in Rhesus monkeys following different regimens of infection. Ann. Trop. Med. Parasitol., 1971, $65,3,367-384$.

Hunter G. W., Crandall R. B., Zickafoose D.E., Purvis O. B.: Studies of Schistosomiasis, XVIII. Some factors affecting resistance to Schistosoma mansoni infections in albino mice. A. J. Trop. Med. Hyg., 1962, 11, 17-24.

Long E., Doenhoff M., Bain J.: Factors affecting the acquisition of resistance against Schistosoma mansoni in the mouse. II. The time at which resistance to re-infection develops. J. Helm., 1978, 52, 187-191.

Michael A. I., Awadalla H. N., Farag H.F.: Granuloma size in the liver of mice with Schistosoma haematobium infection and Schistosoma mansoni challenge. Tropenmed. Parasit., 1979, 30, 62-64.

Pelley R.P., Warren K. S. : Immuno-regulation in chronic infections diseases : Schistosomiasis as a model. J. Invest. Derm., 1978, 71, 49-55.

Ritchie L.S., Knight W. B., Mc Mullen D. B., von Lichtenbergh F.: The influence of infection intensity of Schistosoma mansoni in resistance against existing and subsequent infection in Macaca Mulatta. Am. J. Trop. Med. Hyg., 1966, 15, $43-48$. 
Sher A., Mackenzie P., Smithers S. R.: Decreased recovery of invading parasites from the lungs as a parameter of acquired immunity to Schistosomiasis in the mouse. J. Infec. Dis., 1974, $130,6$.

Sher A.: Immunity against Schistosoma mansoni in the mouse. Am. J. Trop. Med. Hyg., 1977, $26,6,20-28$.

Smith M. A., Clegg J.A. : Different levels of immunity to Schistosoma mansoni in the mouse: The role of variant cercariae. Parasitology, 1979, 78, 311-321.

Smithers S. R., Terry R. J.: The immunology of Schistosomiasis. Adv. Paras., 1976, 14, 399-422.

Von Lichtenberg F.: Host response to eggs of Schistosoma mansoni: granuloma formation in the unsensitized laboratory mouse. Am. J. Pathol., 1962, 41, 771-781.

Von Lichtenberg F.: Studies on granuloma formation antigen, sequestration and destruction in the Schistosoma pseudo tubercle. Am. J. Pathol., 1964, 45, 75-94.

Warren K.S., Domingo E. O.: Granuloma formation around Schistosoma mansoni, S. haematobium and $S$. japonicum eggs; size and rate of development; cellular composition; cross-sensitivity and rate of egg destruction. Am. J. Trop. Med. Hyg., 1970, 19, 292.

Warren K.S.: The immunopathogenesis of Schistosomiasis a multidisciplinary approach. Trans. Roy. Soc. Trop. Med. Hyg., 1972, 66, 417.

Warren K.S. : Modulation of immunopathology in Schistosomiasis. Ciba Foundation Symposium, New series, 1974, 25, 243-261.

Warren K.S., Pelley R.P., Mahmoud A. A. F. : Immunity and immunopathology following re-infection of mice cured of chronic Schistosomiasis mansoni. Am. J. Trop. Med. Hyg., 1977, 26, 957-962.

Warren K.S.: The pathology, pathobiology and pathogenesis of Schistosomiasis. Nature, London, $1978,273,609-612$. 